\title{
Rare presentation of Crohn's Disease: Massive lower gastrointestinal bleeding
}

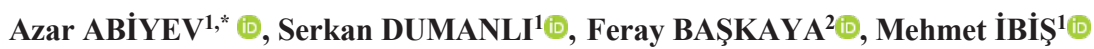 \\ ${ }^{1}$ Department of Gastroenterology, Faculty of Medicine, Gazi University, Ankara, Turkey \\ ${ }^{2}$ Department of Internal Medicine, Faculty of Medicine, Gazi University, Ankara, Turkey
}

\begin{abstract}
\begin{tabular}{ccccc}
\hline Received: 01.02 .2021 & $\bullet$ & Accepted/Published Online: 14.02 .2021 & $\bullet$ & Final Version: 23.04 .2021 \\
\hline
\end{tabular}
\section{Abstract}

While mild gastrointestinal bleeding is common in Crohn's disease, massive gastrointestinal bleeding is extremely rare. In this case, we present a Crohn's patient who presented with massive lower gastrointestinal bleeding. A literature review of such a rare presentation in Crohn's disease will be discussed.
\end{abstract}

Keywords: Crohn's disease, bleeding, endoscopy, inflammation

\section{Introduction}

Crohn's disease (CD) is a chronic inflammatory disease that can affect any part of the gastrointestinal tract from mouth to anus (Veauthier and Hornecker, 2018). Common intestinal complications of Crohn's disease are bleeding, fistula, obstruction and malabsorption

Severe lower gastrointestinal bleeding is a rare but life threatening complication of Crohn's disease. In this setting, bleeding is usually originate from ulcerated areas in colon or intestines (Cirocco et al., 1995). Treatment of the CD is important for mucosal healing and preventing recurrent bleeding. We present a case with massive gastrointestinal bleeding diagnosed with Crohn's disease and successfully treated with prednisolone and azathioprine.

\section{Case report}

A 21 years old male patient referred to our clinic with lower gastrointestinal bleeding and syncope. The bleeding was first started 14 days ago and he admitted to an emergency department. As hemoglobin levels decreased from $13 \mathrm{~g} / \mathrm{dl}$ to $8 \mathrm{~g} / \mathrm{dl}$, he was given three units of erythrocyte suspension. After ressuscitation, esophagogastroduodenoscopy (EGD) and colonoscopy were performed. Both tests were normal and no signs of active bleeding observed. In follow up, hemoglobin levels were stable and bleeding did not recur. So, he was discharged with advice to contact with an advanced center to investigate the bleeding focus.

At the admission, there were no complaints except for mild weakness and occasional constipation. He was not defining any abdominal pain, diarrhea or weight loss. In the past medical history, there was no chronic illness, medication, smoking or alcohol use. Family history was only significant for Crohn's disease in a cousin. There was no lymphadenopathy or organomegalia in physical examination. Rectal examination was also normal. Complete blood count, metabolic panel and liver function tests were performed. Hb level was $12.5 \mathrm{~g} / \mathrm{dl}$ and hematocrit level was 36\%. Erythrocyte sedimentation (ESR) rate and C-reactive protein (CRP) levels were slightly elevated (ESR was $30 \mathrm{~mm} / \mathrm{h}$ and CRP was $10 \mathrm{mg} / \mathrm{dl}$ ). Other tests were normal. Stool examinations were also performed. Microscopic examination and stool cultures were normal. Fecal occult blood test was positive for blood. Fecal calprotectin level was 100 $\mu \mathrm{g} / \mathrm{g}$.

Capsule endoscopy (CE) was performed as the bleeding site could not be detected by EGD and colonoscopy. In the capsule imaging, edematous mucosa with erosions and oozing hemorrhage were seen in the terminal ileum (Fig. 1). Later, colonoscopy and abdominal ultrasonography (USG) were performed to understand the extent of the disease and to take samples for pathologic examination. In the USG, $20 \mathrm{~cm}$ segment wall thickening was observed in proximal terminal ileum. In the colonoscopy, a millimeter-sized ulcer was observed on ileocecal valve. Terminal ileum through the ileocecal valve to proximal $50 \mathrm{~cm}$ was examined. Distal $40 \mathrm{~cm}$ segment of the ileum mucosa was edematous with erosions and milimetric ulcers (Fig. 2). Hypertrophic villis, exudation and "micro cobblestone sign" was prominent in some areas. Multiple samples were taken for tuberculosis and histological examination. Active chronic ileitis with focal involvement and no granuloma was shown in the pathologic examination. Quantiferon test was also performed to exclude tuberculosis 
and it was negative. The findings were consistent with the CD. Prednisolone $40 \mathrm{mg} /$ day and azathioprine (AZA) 50mg/day treatment was started. In two months of follow up, bleeding did not recur and hemoglobin levels were normal.

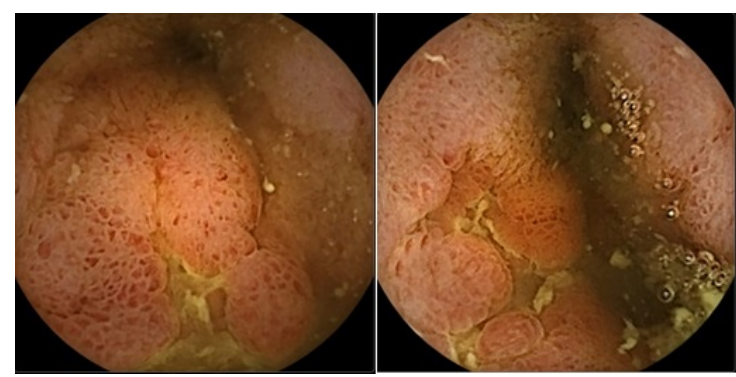

Fig. 1. Capsule endoscopy images

\section{Discussion}

Presentation with massive bleeding in $\mathrm{CD}$ is rare and is detected in only $0.9 \%$ to $2.5 \%$ of cases (Cirocco et al., 1995). Bleeding due to Crohn's disease was first reported in 1941 (Fallis, 1941). In CD with isolated small intestine involvement, the bleeding rate is $23.5 \%$, while in cases with colon involvement, the bleeding frequency is higher (85\%) (Belaiche et al., 1999).

Massive gastrointestinal bleeding is defining as clinical evidence of (1) hemorrhagic shock, (2) syncope related to hematemesis or melena, (3) continued blood loss during the period of one week at no time sufficient to cause syncope or shock but enough to cause a hematocrit reading below $25 \%$ and a hemoglobin concentration below $8 \mathrm{~g} / \mathrm{dl}$ (Atik and Simeone, 1954). So that, hemoglobin decrement of the patient with syncope in the presentation is defined as massive gastrointestinal bleeding. In our patient, there was a syncope history with gastrointestinal bleeding and hemoglobin decrease at the first admission.

Source of bleeding site in our case was not found with EGD and colonoscopy at first. CE is a diagnostic option when the source of bleeding was not found with EGD and colonoscopy have been negative (Pasha et al., 2009). We found the bleeding site in the terminal ileum with capsule endoscopy. Later, we evaluated the ileum with abdominal USG. USG allows early determination of intestinal complications such as abscesses and stenosis, as well as determining the location and size of lesions in CD (Calabrese, 2011). We detected wall thickening in a terminal ileum with USG. We repeated the colonoscopy in order to evaluate this site, as it was possible to reach it by colonoscopy. Colonoscopy findings were consistent with Crohn's disease. Biopsy from the ileum revealed active chronic ileitis with focal involvement, and the patient was diagnosed with Crohn's disease. As they are in the differential diagnosis of the $\mathrm{CD}$, tuberculosis is excluded with negative quantiferon test, intestinal infections are excluded by stool microscopy and culture. There was also no history of non-steroid antinflammatory drug use and no sign of rheumatologic disease in questionnaire and physical examination.
Corticosteroids, azathioprine, and infliximab can be used to reduce the risk of acute bleeding for $\mathrm{CD}$ with acute lower gastrointestinal bleeding (Barnacle et al., 2006). Besides medical treatment, embolization therapy can also be effective (Lee et al., 2020). Surgical resection can be performed in hemodynamically unstable patients who are unresponsive to medical treatment and embolization (Alos and Hinojosa, 2008). The recurrence rates of severe bleeding have been reported from 19\%-41\% (Podugu et al., 2016). In this case, the patient will be followed up with AZA treatment.

In conclusion, $\mathrm{CD}$ presenting with massive gastrointestinal bleeding is very rare. It should be kept in mind that Crohn's disease may cause massive bleeding in young patients, alongside diseases such as Meckel diverticulitis, tumors, Dieulafoy lesion, and polyposis. USG, which is well accepted by patients, noninvasive, and low cost, may be the first diagnostic tool, especially in young patients, as it can be used for preliminary diagnostic work-up prior to invasive tests

\section{Conflict of interest}

None to declare.

\section{Acknowledgments}

None to declare.

\section{References}

1. Alós R, Hinojosa J., 2008. Timing of surgery in Crohn's disease: a key issue in the management. World J. Gastroenterol. 14(36), 5532-5539.

2. Atik, M., Simeone, F.A., 1954. Massive gastrointestinal bleeding: study of two hundred ninety-six patients at city hospital of cleveland. AMA Arch. Surg. 69(3), 355-365.

3. Barnacle, A.M., Aylwin, A.C., Jackson, J.E., 2006. Angiographic diagnosis of inflammatory bowel disease in patients presenting with gastrointestinal bleeding. AJR. Am. J. Roentgenol. 187, 976-985.

4. Belaiche, J., Louis, E., D'Haens, G., et al., 1999. Acute lower gastrointestinal bleeding in Crohn's disease: Characteristics of a unique series of 34 patients. Belgian IBD Research Group. Am. J. Gastroenterol. 94(8), 2177-2181.

5. Cirocco, W.C., Reilly, J.C., Rusin, L.C., 1995. Life-threatening hemorrhage and exsanguination from Crohn's disease. Report of four cases. Dis. Colon. Rectum. 38, 85-95.

6. Fallis, L.S., 1941. Massive intestinal hemorrhage in regional enteritis: report of a case. Am. J. Surg. 53, 512-513.

7. Lee, K.C., Kuo, C.J., Tseng, J.H., et al., 2020. The presentation of Crohn's disease with acute massive gastrointestinal hemorrhage. Adv. Dig. Med. 1-4.

8. Pasha, S.F., Hara, A.K., Leighton, J.A. 2009. Diagnostic evaluation and management of obscure gastrointestinal bleeding: a changing paradigm. Gastroenterol. Hepatol. 5(12), 839-50.

9. Podugu, A., Tandon, K., Castro, F.J., 2016. Crohn's disease presenting as acute gastrointestinal hemorrhage. World J. Gastroenterol. 22(16), 4073-4078.

10. Veauthier, B., Hornecker, J.R., 2018. Crohn's Disease: Diagnosis and Management. Am. Fam. Physician. 98(11), 661669. 